\title{
Concurrent Vitiligo and Psoriasis
}

\author{
Karn D, KC S
}

\author{
Department of Dermatology \\ Kathmandu University School of Medical Sciences \\ Dhulikhel Hospital, Kathmandu University Hospital \\ Dhulikhel, Nepal
}

Corresponding Author

Dharmendra Karn

Department of Dermatology

Kathmandu University School of Medical Sciences

Dhulikhel Hospital, Kathmandu University Hospital

Dhulikhel, Nepal

E-mail: dddkarn@gmail.com

\begin{abstract}
Coexistence of vitiligo and psoriasis in a single patient is a rare occurrence. The exact pathogenesis of this coexistence is obscure. Various autoimmune disorders are reported to occur more frequently in patients with both diseases. A case of this interesting coexistence seen on a 26 year old female patient who presented to the dermatology department of Dhulikhel hospital is presented here. She also had hypothyroidism. The possible pathogenesis and literature review of this rare condition is discussed here.
\end{abstract}

\section{KEYWORDS}

Vitiligo, psoriasis, autoimmune disorders, hypothyroidism

\section{Citation}

Karn D, KC S. Concurrent Vitiligo and Psoriasis. Kathmandu Univ Med J 2012;37(1):83-4.

\section{INTRODUCTION}

Psoriasis and Vitiligo are relatively common diseases affecting $0.5 \%$ and $1-3 \%$ of the general population respectively. ${ }^{1}$ Although immunologic phenomena is considered in the pathophysiology of both diseases, their coexistence is unusual. ${ }^{2,4}$ The first case of psoriasis with vitiligo was reported by Selenyi in $1955 .^{3}$ These coexistence are often thought to be associated with underlying autoimmune conditions. We are reporting here a successfully managed case of concurrent vitiligo and psoriasis which also highlights the possible link between the pathogenesis of the two conditions.

\section{CASE REPORT}

A 26 year old female presented to the Dermatology department of Dhulikhel Hospital with complaints of red, Itchy and scaly skin lesions for past two years. She had also multiple white lesions more on her back, abdomen and lower extremities. This lesion was diagnosed as vitiligo 22 years back and has been not treated so far. The scaly lesions were initially confined to the depigmented areas but now have spread to normal skin as well. She had a family history of similar depigmented lesions on her mother. On systemic review, she was often constipated but had no other systemic complaints.

On examination she had multiple depigmented macules and ill defined, erythematous plaques with silvery white scales with positive Auspitz sign. ${ }^{5}$

A clinical diagnosis of coexisting vitiligo with unstable plaque psoriasis was made. Individual skin biopsies were taken from three sites: vitiligo, psoriasis and from psoriasis superimposed on vitiligo. Histopathology was suggestive of psoriasis but due to the absence of special staining for melanocyte, pathological diagnosis of vitiligo could not be made.Blood investigations were as Total count (12100/ $\mathrm{mm}$ ), differential count (neutrophil-78\%, lymphocyte-19\%, eosinophil-3\%), hemoglobin (11.5gm\%), erythrocyte sedimentation rate ( $27 \mathrm{~mm}$ in first hour, Wintrobe method) and random blood sugar $(86 \mathrm{mg} / \mathrm{dl}) .^{3}$ Lipid profile, liver function test, renal function test and electrocardiography were normal. Thyroid function test revealed hypothyroidism with freeT3-2.05 picogram/ml; free T4-0.5 nanogram/ 


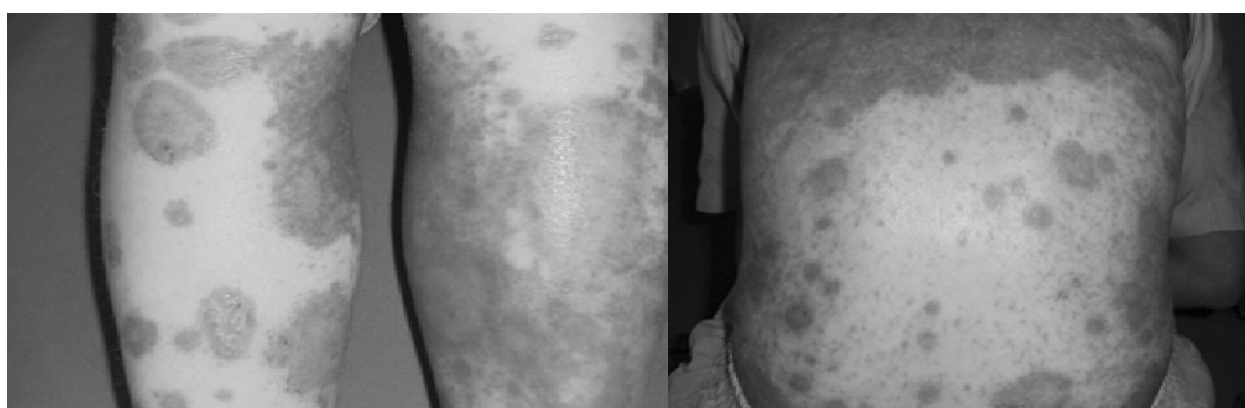

Figure 1. Vitiligo with psoriasis on patient's legs and back.

$\mathrm{ml}$ and TSH-44.05 micro international unit/ml. She had positive antinuclear antibody with a titre of 2.30. However, Rheumatoid factor was negative.

Patient was admitted and was started on specific T-cell inhibitor: cyclosporine $50 \mathrm{mg} /$ day, thyroxine, supportive therapy followed by Acitretin $25 \mathrm{mg}$ twice daily and Psoralen and Ultraviolet A (PUVA) therapy. There was expected improvement in psoriasis and was discharged then with treatment for vitiligo. She is now on regular follow up with good results for vitiligo as well.

\section{DISCUSSION}

Psoriasis is a chronic, non infectious, inflammatory disease of the skin characterized by well defined erythematous plaques with large, adherent silvery scales. ${ }^{6}$ Vitiligo leads to depigmentation of skin, in which some or all melanocytes in the interfollicular epidermis and occasionally those in the hair follicles are selectively destroyed. Despite being common their coexistence is rarely seen. The pathogenic mechanism for this coexistence is not completely clear and there have been only few cases reported in the literature. ${ }^{4}$

Vitiligo based on distribution pattern may be generalized, localized or universal. Generalized type of vitiligo includes segmental, vulgaris and mixed types. ${ }^{6}$ In terms of classification, this patient falls under generalized (mixed) type and she had unstable plaque psoriasis. Vitiligo is frequently found to be associated with thyroid dysfunction. $39.4 \%$ of patients were found to have abnormal thyroid function test especially hypothyroidism. ${ }^{5}$

Based on a retrospective study on 712 patients with vitiligo [446 female (63\%) and 266 male (37\%)\}, 21 (3\%) of them had associated psoriasis [11 male and 10 female]. ${ }^{7}$ Vitiligo was generalized in 16 cases and psoriasis was psoriasis vulgaris in 18 patients. Co morbidities were present among nine patients: arthritis in two, prostatic hypertrophy in two, allergic rhinitis in two, hypertension in one and thyroid disorder in two. Including autoimmune thyroiditis and arthritis a total of four patients had associated autoimmune disease.

Both disease can have an autoimmune component in their pathogenesis and can be associated with other autoimmune states. Further, Tumor Necrosis Factor (TNF)alpha is a major cytokine elevated in psoriatic lesions and an increased levels of TNF-alpha have also been found in the perilesional skin in patients with vitiligo. ${ }^{6,8}$ So, TNF-alpha may represent a common link between vitiligo and psoriasis and needs further evaluation to explore the pathogenetic mechanism for these conditions. This coexistence is also a possible result of T- lymphocyte mediated autoimmunity because immunosuppression of T- lymphocytes with drugs as cyclosporine results in rapid improvement of the condition. Thyroid disease, arthritis, bullous pemphigoid, alopecia areata, pemphigus vulgaris, lichen planus and pernicious anemia are considered some associations in patients with coexisting vitiligo and psoriasis.

There is very limited number of such cases presenting to us and there are limited case reports in literature as well. It remains as our limitation for the study of its underlying pathogenesis.

\section{CONCLUSION}

Coexistence of vitiligo and psoriasis in a single patient is a rare occurrence. An underlying autoimmune condition has to be sought for when such coexistence is seen. There is a need of increasing case report of such a rare coexistence in literature so that their basic underlying pathogenesis can be explored.

\section{REFERENCES}

1. Park JM, Kim HJ, Bae BG, Park YK. A case of concurrent vitiligo and psoriasis. Annals of dermatology. 2009; 21(3):330-3.

2. Inamadar AC, Sampagavi VV, Athanikar SB, Patil MN, Deshmukh NS. Vitiligo and psoriasis: Coexistence with colocalisation. Indian $J$ Dermatol Venereol Leprol 2001; 67:214-5.

3. Selenyi A. Vitiligo and psoriasis on the same side with syringomyelia. Borgyogy Venerol Sz. 1955; 9:94-96.

4. Pasic A, Ljubojevic S, Lipozencic J, Marinovic B, Loncaric D. Coexistence of psoriasis vulgaris, bullous pemphigoid and vitiligo: a case report. $J$ Eur Acad Dermatol Venerol 2002; 16:426-7.

5. Subba K, Karn D, Khatri R. Triiodothyronine, thyroxine and thyrotropin in vitiligo. Kathmandu Univ Med J 2011; 34(2):7-10.

6. Burns $T$, Breathnach S, Cox N, Griffiths C. Disorders of Skin colour. Rook's textbook of dermatology. 8th ed. Wiley-Blackwell; 201:5.18.

7. Sandhu K, Kaur I, Kumar B. Psoriasis and vitiligo. J Am Acad Dermatol. 2004;51:149-150.

8. Percivalle S, Piccinno R and Caccialanza M. Concurrence of vitiligo and psoriasis: a simple coincidence? Clin and Exp Dermatol. 2009;34:90-1. 